\title{
Magnetic Resonance Imaging of Intracavernous Pathology
}

\author{
Masaki KomiYama, Akira HakuBA*, Toshihiro Yasul, \\ Hisatsugu YAGURA, Yoshihiko FU, Mitsuru BABA \\ and Shuro NISHIMURA*
}

Department of Neurosurgery, Baba Memorial Hospital, Sakai, Osaka;

*Department of Neurosurgery, Osaka City University Medical School, Osaka

\begin{abstract}
To evaluate the usefulness of magnetic resonance (MR) imaging of intracavernous pathology, $T_{1}$ weighted spin echo images of four vascular lesions and 10 neoplastic lesions with surgically confirmed cavernous sinus (CS) invasion were reviewed retrospectively. In one case of traumatic carotid-cavernous fistula (CCF) and one of dural arteriovenous malformation (AVM), the internal carotid artery (ICA) and rapid shunted flow were depicted as signal voids, and the relationship between the ICA and shunted flow was clearly shown. Normal venous flow appeared as a low-intensity area and was observed even in the presence of the CCF and dural AVM. In two cases of thrombosed aneurysms, the thrombosis was clearly demonstrated, along with patent arterial flow in one case; in the other case, however, it was impossible to differentiate patent arterial flow from calcification. The intensity of all neoplastic lesions was similar to that of the cerebral cortex. The relationship between the ICA and the tumors was clearly demonstrated. The visual pathways were also plainly shown unless they were involved, or markedly compressed, by tumor. CS invasion was strongly associated with four findings: 1) encasement of the ICA by the tumor; 2) marked displacement of the ICA; 3) absence of low intensity, which reflects normal venous flow, in the CS; and 4) extension of extrasellar tumors to the medial wall or of intrasellar tumors to the lateral wall. MR imaging was judged promising in the evaluation of intracavernous pathology.
\end{abstract}

Key words: cavernous sinus, magnetic resonance imaging, vascular lesion, neoplastic lesion

\section{Introduction}

The cavernous sinus (CS) is a complex structure, and many detailed studies of its anatomy have been performed..$^{2,5,10,12,15)}$ Demonstration of intracavernous pathologies is very important in designing a therapeutic plan. However, recent advances in vascular surgery and direct microsurgical manipulation in the CS require increasingly detailed anatomical information. In cases of vascular lesions, demonstration of the internal carotid artery (ICA), normal venous flow, rapidly flowing shunted blood, if any, and the anatomical relationship with surrounding structures is extremely important. Similarly, invasion of the CS and the relationship to the visual pathways in cases of neoplastic lesions are of the

Received January 25, 1988; Accepted March 29, 1989 utmost concern.

The usefulness of magnetic resonance (MR) imaging in the evaluation of various central nervous system lesions is well recognized. The aim of this study was to determine the usefulness of MR imaging in the evaluation of intracavernous pathology.

\section{Patients and Methods}

MR images of 14 patients with intracavernous pathology of various etiologies were retrospectively reviewed. On the basis of their clinical diagnoses, the patients were divided into two groups (Table 1). Group A included four patients with vascular lesions: one traumatic carotid-cavernous fistula (CCF), one dural arteriovenous malformation (AVM), and two giant thrombosed aneurysms. The two males and two females in this group ranged from 18 to 49 years of age. Group B comprised 10 patients with 
Table 1 Summary of 14 cases of intracavernous pathology

\begin{tabular}{cccc}
\hline Case No. & Age & Sex & \multicolumn{1}{c}{ Diagnosis } \\
\hline \multicolumn{3}{c}{ Group A: vascular lesions } & \\
1 & 18 & M & traumatic CCF \\
2 & 45 & M & dural AVM \\
3 & 45 & F & thrombosed aneurysm \\
4 & 49 & F & thrombosed aneurysm
\end{tabular}

Group B: tumors invading the CS

$\begin{array}{rrrl}5 & 35 & \text { M } & \text { meningioma } \\ 6 & 49 & \text { M } & \text { meningioma } \\ 7 & 56 & \text { M } & \text { meningioma } \\ 8 & 34 & \mathrm{~F} & \text { neurinoma } \\ 9 & 48 & \mathrm{~F} & \text { neurinoma } \\ 10 & 17 & \mathrm{~F} & \text { pituitary adenoma } \\ 11 & 23 & \mathrm{~F} & \text { pituitary adenoma } \\ 12 & 37 & \mathrm{~F} & \text { pituitary adenoma } \\ 13 & 39 & \mathrm{~F} & \text { pituitary adenoma } \\ 14 & 59 & \mathrm{~F} & \text { chondroma }\end{array}$

neoplastic lesions: three meningiomas, two neurinomas, four pituitary adenomas, and one chondroma. The ages of these three males and seven females ranged from 17 to 59 years.

MR examinations were performed with a $0.5-\mathrm{T}$ superconductive imager (Vista-MR scanner, Picker International, Cleveland, Ohio, USA). The pulse sequences were $T_{1}$-weighted single spin echo ( $\mathrm{SE}$ ), the echo and repetition times being 30-40 and 600$1000 \mathrm{msec}$, respectively. The image planes were orthogonal, with a thickness of 0.5 or $1.0 \mathrm{~cm}$. The field of view was $30 \mathrm{~cm}$ and the image matrix $256 \times$ 256; the number of slices imaged simultaneously was 1,4 , or 8 . The data were averaged once. In selected cases, gadolinium-diethylenetriaminepentaacetic acid (Gd-DTPA) was administered at a dose of $0.1 \mathrm{mmol} / \mathrm{kg}$ body weight injected over 1 minute and postcontrast scans were obtained within 40 minutes of its administration. In all cases, plain skull $\mathrm{x}$-rays, pre- and postcontrast computed tomographic (CT) scans, and cerebral angiograms were obtained. All vascular lesions were diagnosed radiologically. All neoplastic lesions were diagnosed histologically and CS invasion was verified surgically.

\section{Results}

\section{Group A: vascular lesions}

Traumatic CCF (Fig. 1): The ICA and rapidly flowing shunted blood in the draining channels within the CS were visualized as signal voids, whereas the remaining venous flow, even on the ipsilateral side of
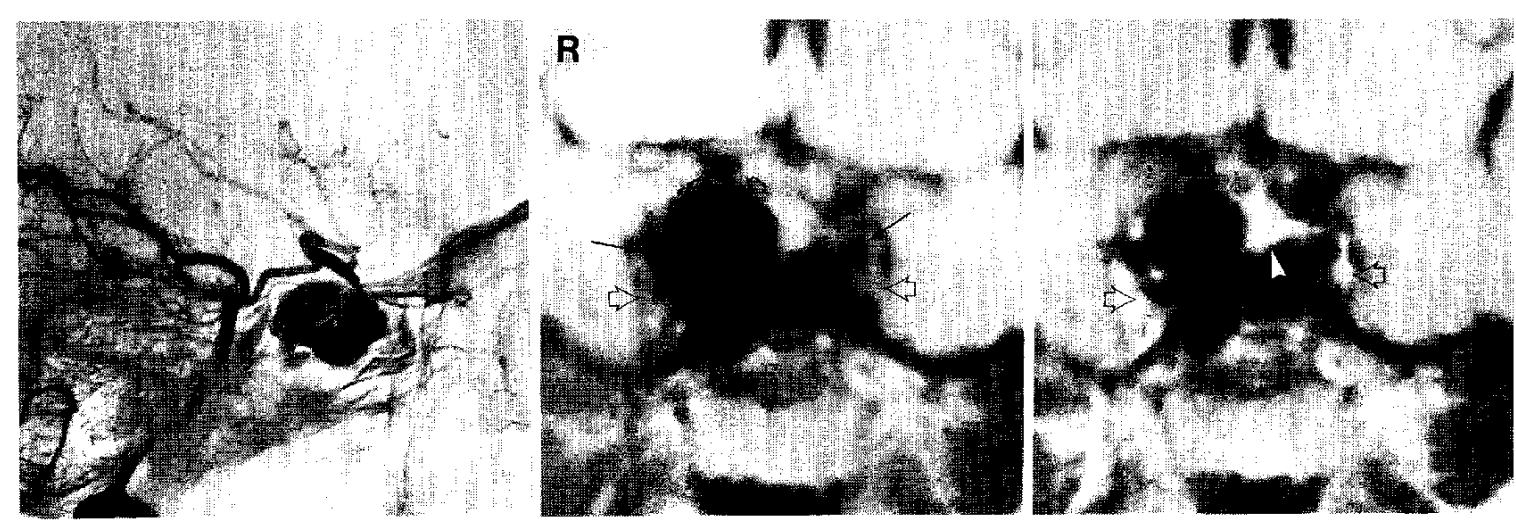

Fig. 1 Case 1. Traumatic CCF. left: Right vertebral angiogram showing retrograde demonstration of the $\mathrm{CCF}$ through the posterior communicating artery and the distal ICA. The middle cerebral artery is also faintly visualized. It is difficult to identify the intracavernous portion of the ICA and to appreciate the relationship between the ICA and the fistula. Right carotid angiography (not shown) also failed to disclose their relationship. center: $\mathrm{T}_{1}$-weighted $\mathrm{SE}$ image without $\mathrm{Gd}$ DTPA. The ICAs (arrows) and a large draining channel (hatched arrows) appear as signal voids due to high-velocity signal loss. Although the site of the fistula cannot be identified, the anatomical relationship between the ICA and the draining channel is clearly shown. Note the bilateral lowintensity areas of normal venous flow (open arrows) in the CS. right: $\mathbf{T}_{1}$-weighted SE image enhanced with Gd-DTPA. The ICAs and the large draining channel remain devoid of signals even after administration of Gd-DTPA. However, Gd-DTPA markedly enhances normal venous flow (open arrows) in the CS. The pituitary gland (arrowhead) is also enhanced with Gd-DTPA. In this way, Gd-DTPA is helpful in identifying normal venous flow. 

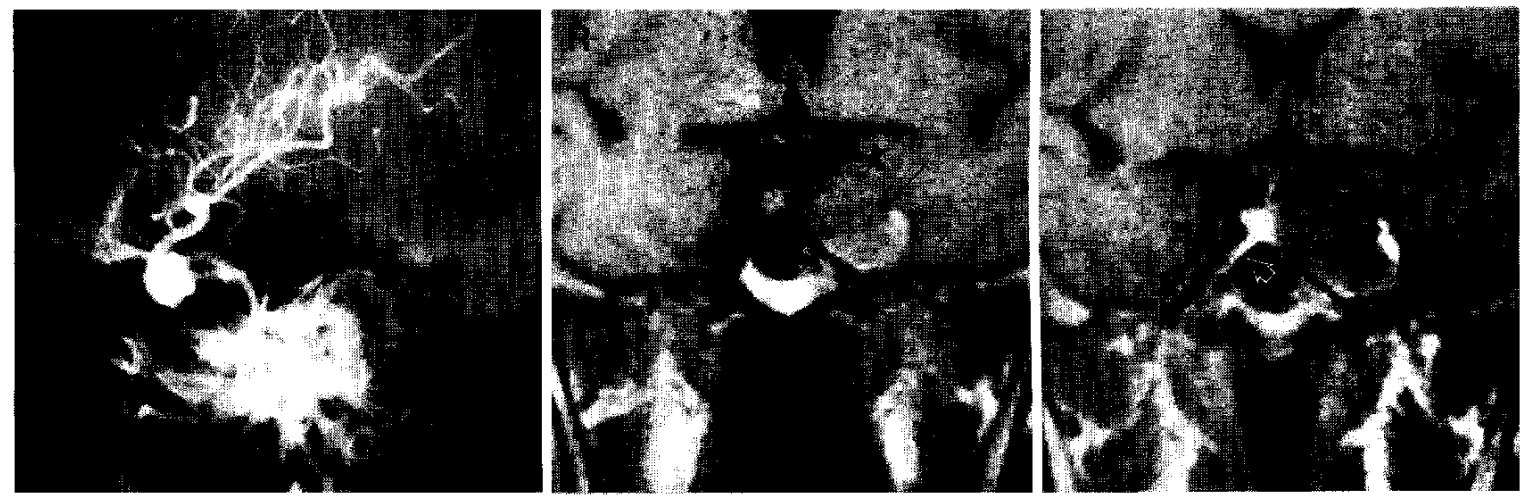

Fig. 2 Case 4. Thrombosed aneurysm. left: Left carotid arteriogram showing the patent lumen within a giant aneurysm. center: $T_{1}$-weighted SE image without Gd-DTPA. The thrombus (arrowhead) exhibits almost the same intensity as the cerebral cortex. The right ICA and the patent carotid flow (arrow) within the thrombus appear as signal voids. Inferior to the patent artery on the left, a small high-intensity area (hatched arrow) represents methemoglobin within the clot. right: $\mathrm{T}_{1}$-weighted SE image enhanced with Gd-DTPA. The right ICA and the carotid blood flow within the thrombus remain signal voids, whereas the scant venous flow (thick arrow) immediately lateral to the arterial flow is markedly enhanced. This high-intensity area is not seen in Fig. 2 center. Normal venous flow on the right (open arrow) and the pituitary gland are also enhanced with Gd-DTPA. The highintensity area (hatched arrow) represents methemoglobin within the clot.

the CCF, appeared as an area of low signal intensity. Gd-DTPA markedly enhanced the remaining venous flow, but enhanced neither arterial flow in the ICA nor shunted flow in the draining channels. The dilated superior ophthalmic vein also appeared as a signal void. Although the site of the fistula was not precisely delineated, the relationship between the ICA and draining channels was clearly shown, especially on the coronal images.

Dural AVM: The AVM itself, which was demonstrated by cerebral angiography, could not be identified with MR imaging. The dilated draining channels in the CS were represented by signal voids immediately lateral to the ICA, while the remaining venous channels were visualized as areas of low intensity. The dilated superior ophthalmic vein also appeared as a signal void.

Giant thrombosed aneurysms (Fig. 2): In one case (Case 4), patent arterial flow in the thrombosis was displayed as a signal void and a thrombosed clot as a low-intensity area, the intensity resembling that of cerebral cortex. The CS was almost obliterated by the clot; only a small residual venous space remained at the lateral wall of the thrombosed aneurysm, and this space was markedly enhanced with GdDTPA. High intensity in an area just inferior to this venous space on both pre- and postcontrast images was due to methemoglobin within the clot.

In the second case (Case 3), the wall of the aneurysm showed a signal void, which corresponded with the calcification depicted by CT. Small patent arterial flow was not definitely depicted by MR imaging since it was too small, with calcification located very near to it, showing similar intensity. The CS was completely obliterated by the thrombosis, leaving no venous flow. The clot exhibited two intensities, one similar to that of cerebral cortex and the other much lower.

\section{Group B: neoplastic lesions (Figs. 3-5)}

On $T_{1}$-weighted SE images, all tumors showed approximately the same intensity as the cerebral cortex. The relationship between the visual pathways and the tumor was clearly depicted unless the visual pathways were involved by the tumor or were markedly compressed. The relationship between the ICA, anterior cerebral arteries, and tumor was also clearly shown because arterial flow always appeared as a signal void.

Encasement of the ICA by the tumor was recognized in six of the 10 cases, and marked displacement of the ICA in five cases. Absence of low signal intensity, representing normal venous flow, in the invaded CS was noted in all cases but one (chondroma). This was usually observed on most coronal images but was difficult to appreciate on axial or sagittal images. Extension of intrasellar tumors to the lateral wall was noted in four cases of pituitary adenoma and extension of extrasellar tumors to the medial wall was noted in four other cases. 


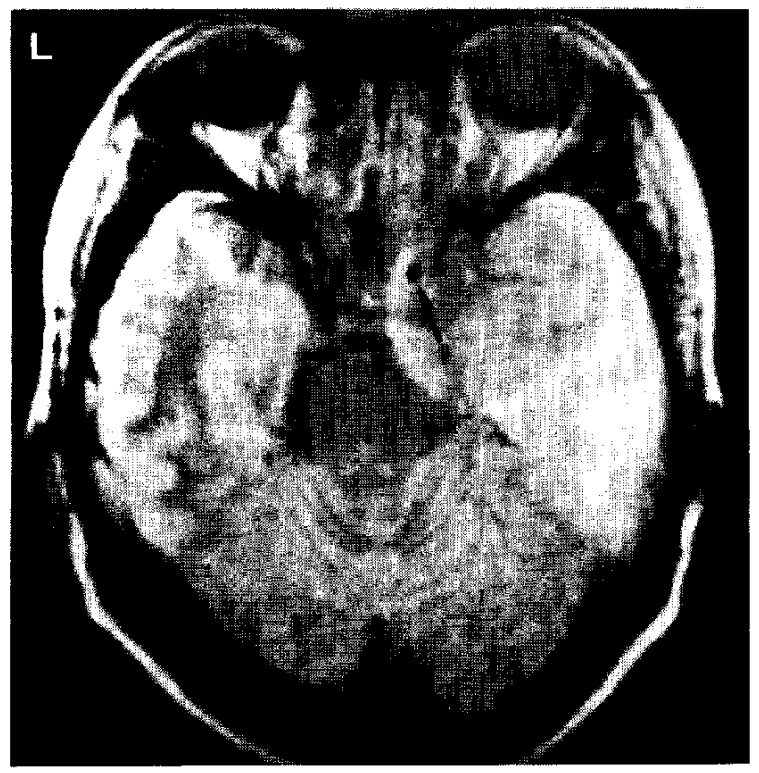

Fig. 3 Case 6. Meningioma with invasion of the right CS. On this $T_{1}$-weighted $\mathrm{SE}$ image without Gd-DTPA, a signal void representing the ICA (arrow) is completely engulfed by tumor and markedly displaced anteromedially. It is usually difficult to identify normal venous spaces on axial images.

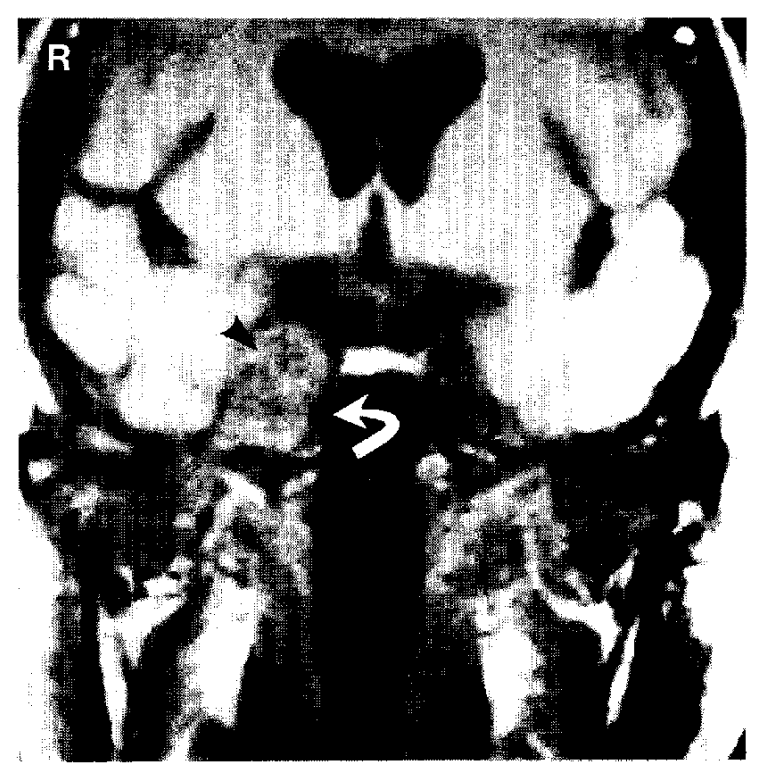

Fig. 4 Case 9. Neurinoma with invasion of the right CS. On a $\mathrm{T}_{1}$-weighted SE image without GdDTPA, the neurinoma (arrowhead) exhibits heterogeneous low intensity and partially encases the ICA. The vertical portion of the ICA is partially visualized (curved arrow). The low intensity that would reflect normal venous flow is absent on the right.

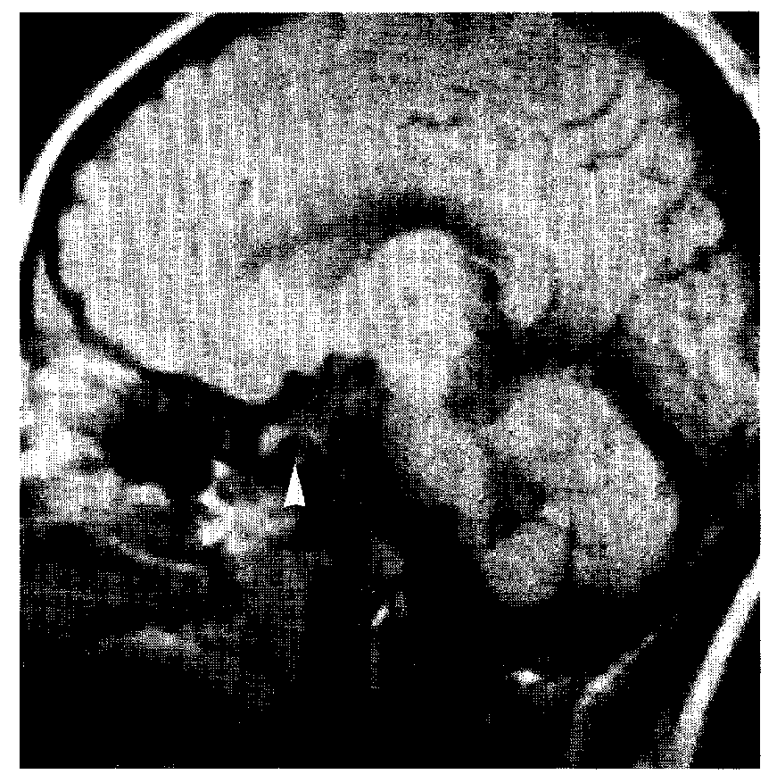

Fig. 5 Case 10. Pituitary adenoma with invasion of the left CS. A $T_{1}$-weighted SE image of the left parasagittal plane without Gd-DTPA demonstrates the left ICA as a signal void and shows the intracavernous ICA to be encased by tumor (arrowhead).

\section{Discussion}

The increasing capability of direct surgical intervention in the CS necessitates precise knowledge of this structure, not only of its anatomy but also of blood flow, since the CS contains both important neural and vascular components.

MR imaging demonstrates two principal flowrelated phenomena: paradoxical enhancement (flowrelated enhancement and even-echo rephasing) and high-velocity signal loss. ${ }^{3)}$ Paradoxical enhancement, which increases signal intensity, represents slow flow, whereas high-velocity signal loss reflects rapid blood flow. The Gd (III) ions in Gd-DTPA reduce the $T_{1}$ and $T_{2}$ of surrounding protons, a phenomenon referred to as "proton relaxation enhancement." 14) This results in high signal intensity on $\mathrm{T}_{1^{-}}$ weighted SE images.

On $\mathrm{T}_{1}$-weighted SE images of a normal CS, blood flow in the ICA is always visualized as a signal void, due to high-velocity signal loss, and is not enhanced with Gd-DTPA. Daniels et $a l^{4)}$ reported that venous blood flowing through the CS produces only negligible signals. In our study, venous flow in the CS, unlike arterial flow, ${ }^{7,8}$ ) was usually demonstrated as areas of low signal intensity rather than as absolute signal voids, probably because slow ve- 
nous flow was subject to paradoxical enhancement. The venous spaces were markedly enhanced with Gd-DTPA. In contrast to the CS, the superior sagittal and transverse sinuses were usually visualized as signal voids, due to rather rapid blood flow. Rapidly flowing shunted blood in cases of CCF and dural AVM also appeared as signal voids because of high-velocity signal loss.

In traumatic CCF, cerebral angiography clearly demonstrates draining channels in the CS, but sometimes fails to disclose the site of the fistula itself. In our case, MR images clearly showed the ICA and the dilated draining channels and their relationship. Mullan ${ }^{11)}$ described two compartments of CS divided by the ICA: a larger, anteroinferior section and a smaller, posterosuperior section. He proposed that, in CCF, distension of a main channel obliterates lesser channels, allowing the two compartments to become contiguous, although they occasionally may remain separate. Harris and Rhoton ${ }^{5)}$ observed three major venous spaces within the CS, posterosuperior, anteroinferior, and medial to the cavernous portion of the ICA, which appeared to be largely unbroken, trabeculated venous channels. Contrary to their observations, in our case MR images clearly demonstrated venous channels that were unrelated to the $\mathrm{CCF}$, and this was confirmed by enhancement with Gd-DTPA. These venous channels probably contained slowly flowing blood, despite the presence of a CCF. This finding supports Parkinson's concept ${ }^{13)}$ of the CS as a plexus of various-sized venous channels. Regarding CCF, it can be inferred that one venous channel is markedly dilated with shunted flow, whereas the remaining channels, which have no direct connection with the CCF, have normal venous flow. Nonshunted blood flow has not been satisfactorily demonstrated with conventional diagnostic modalities. MR imaging provided excellent and separate disclosure of shunted and nonshunted blood flow. This information is helpful in the planning of both surgery and intraluminal procedures for CCF.

Although a dural AVM itself is not readily demonstrated by MR imaging, the draining channels are clearly shown, owing to the high-velocity signal loss of the rapid shunted flow. Kucharczyk et al. ${ }^{\text {9) }}$ reported the failure of MR imaging to disclose cavernous dural AVMs. Although cerebral angiography is the diagnostic modality of choice, MR imaging also has diagnostic value, since a dilated CS, which reflects rapidly flowing shunted blood, can be shown noninvasively and repeatedly. As with $\mathrm{CCF}$, normal nonshunted venous flow was demonstrated by MR imaging, together with the rapid shunted flow in our case.

An aneurysm is usually recognized as a dilated vascular cavity connected to the normal vascular anatomy and appears as a signal void due to highvelocity signal loss. However, paradoxical enhancement may occur in the case of slow flow. Thromboses must be differentiated from neoplasms, but this is sometimes difficult. Methemoglobin and hemosiderin appear, peculiarly, as high and low intensity, respectively, which may contribute to the differentiation of aneurysms from neoplastic lesions. In one of our cases (Case 3), part of the thrombosis exhibited very low intensity, probably due to hemosiderin. Residual venous flow in a CS almost obliterated by an aneurysm, which is rarely demonstrable with cerebral angiography, was visualized in one of our cases of a large, thrombosed aneurysms (Case 4); the scant venous flow was markedly enhanced with Gd-DTPA. In this way, venous flow in the CS can be identified preoperatively.

$\mathrm{T}_{1}$-weighted $\mathrm{SE}$ images provide a high signal-tonoise ratio and thus suitably demonstrate anatomical features. Although they cannot provide high contrast between tumor and normal tissue, they are useful to study the CS because of their high spatial resolution. Due to the relative lack of tissue contrast on $\mathrm{T}_{1}$-weighted $\mathrm{SE}$ images, all tumors in our study showed almost the same intensity as cerebral cortex. However, it is very important to ascertain whether or not the tumor has invaded the CS, since CS invasion sometimes determines whether the therapeutic plan will be radical surgery or palliative radiation. Kline et $a l .{ }^{6}{ }^{6}$ reported that the $\mathrm{CT}$ features suggestive of an abnormal CS are 1) asymmetry of size; 2) asymmetry of shape, with particular reference to the lateral wall; and 3) focal areas of abnormal density within the CS. Ahmadi et al. ${ }^{1)}$ listed the following indications of CS invasion by pituitary adenomas: 1) CS expansion; 2) visible encasement of the ICA; 3) compression or displacement of the intracavernous cranial nerves; 4) invasion of the lateral wall; and 5) diffuse bony destruction. They also noted that MR images demonstrated ICA encasement in three cases.

When the tumor completely encases the ICA, invasion of the CS is obvious. However, complete encasement of the ICA is not always necessary for diagnosing invasion of the $\mathrm{CS}$. We noted four MR findings to be suggestive of CS invasion: 1) partial or complete encasement of the ICA by the tumor; 2 ) marked displacement of the ICA;3) absence of the low signal intensity that represents normal venous flow in the CS and is markedly enhanced with GdDTPA; and 4) extension of an intrasellar tumor to 
the lateral wall or of an extrasellar tumor to the medial wall. Except for the absence of low intensity, these features also apply to CT. The lack of artifacts from adjacent bone is one advantage of MR imaging; CT images of the CS are always degraded by streak artifacts and coronal images can be obtained only with the patient's cooperation. Since the intracavernous cranial nerves were not always visualized with our MR system, we could not use involvement of the cranial nerves as a criterion for determining CS invasion. With further improvement in MR technology, involvement of the cranial nerves will also serve as a useful indicator of CS invasion.

In conclusion, we found that MR imaging provided far more detailed information concerning anatomy and blood flow in the CS than did other diagnostic modalities, including CT. Our preliminary experience encourages our further use of $\mathrm{MR}$ imaging in this field.

\section{References}

1) Ahmadi J, North CM, Segall HD, Zee C-S, Weiss $\mathrm{MH}$ : Cavernous sinus invasion by pituitary adenomas. AJNR 6: 893-898, 1985

2) Bedford MA: The "cavernous" sinus. Brit J Ophthal 50: 41-46, 1966

3) Bradley WG, Waluch V: Blood flow: Magnetic resonance imaging. Radiology 154: 443-450, 1985

4) Daniels DL, Pech P, Mark L, Pojunas K, Williams AL, Haughton VM: Magnetic resonance imaging of the cavernous sinus. AJNR 6:187-192, 1985

5) Harris FS, Rhoton AL: Anatomy of the cavernous sinus: A microsurgical study. I Neurosurg 45: 169 180,1976

6) Kline LB, Acker JD, Post MJD, Vitek JJ: The cavernous sinus: A computed tomographic study. $A J N R$
2: 299-305, 1981

7) Komiyama M, Yasui $T$, Baba M, Hakuba A, Nishimura S, Inoue Y: MR imaging of blood flows in the cavernous sinus. Radiation Medicine 6: 124-129, 1988

8) Komiyama $M$, Yasui $T$, Baba $M$, Hakuba A, Nishimura S, Nemoto Y, Inoue Y: MR imaging: Normal and invaded cavernous sinus studied with and without Gd-DTPA, in Dolenc VV (ed): The Cavernous Sinus. Wien, Springer, 1987, pp 152-161

9) Kucharczyk W, Lemme-Pleghos L, Uske A, BrantZawadzki M, Dooms G, Norman D: Intracranial vascular malformations: $\mathrm{MR}$ and $\mathrm{CT}$ imaging. Radiology 156: 383-389, 1985

10) McGrath P: The cavernous sinus: An anatomical survey. Aust NZ J Surg 47: 601-613, 1977

11) Mullan S: Treatment of carotid-cavernous fistulas by cavernous sinus occlusion. I Neurosurg 50: 131-144, 1979

12) Parkinson D: Collateral circulation of cavernous carotid artery: Anatomy. Canad J Surg 7: 251-268, 1964

13) Parkinson D: Carotid cavernous fistula: Direct repair with preservation of the carotid artery. J Neurosurg 38: 99-106, 1973

14) Runge VM, Clanton JA, Lukehart CM, Partain CL, James AE: Paramagnetic agents for contrast-enhanced NMR image: A review. AJR 141: 1209-1215, 1983

15) Umansky F, Nathan $H$ : The lateral wall of the cavernous sinus: With special reference to the nerves related to it. $J$ Neurosurg 56: 228-234, 1982

Address reprint requests to: M. Komiyama, M.D., Department of Neurosurgery, Baba Memorial Hospital, 4244 Higashi, Hamadera-funao-cho, Sakai, Osaka 592, Japan. 